\title{
The Post \\ Pneumonectomy Chest Radiograph
}

\begin{abstract}
$E_{\text {achyear in South Africa hundreds }}$ of pneumonectomies are performed. In the western world the vast majority are for treatmentoflung carcinoma. Locally, the indications are equally balanced with those for complications of tuberculosis and other inflammatory lung disease. The younger average age of these cases creates a relatively higher prevalence of post pneumonectomy patients in the general population. The spectrum of postoperative changes is broad and the differentiation between what is normal appearance and what is pathological can have a great bearing on therapeutic intervention.
\end{abstract}

\section{Early \\ Postoperative Changes}

\section{Nuances of technique}

The basic technique of pneumonectomy has remained unchanged for over 40 years. In the mid 70s the popularisation of staple closure of the main bronchi offered one of the few improvements in technique and a general reduction in the incidence of the leak. Radiological evidence of staples can be seen on most contemporary radiographs which also act as a convenient marker of stump length and position. The resection of ribs for pleural access is unnecessary with the exception of rare instances of profound pleural thickening or local malignant extension. It is still often seen however where surgery was performed in the preceding decades. It is not rare to sustain rib fractures, typically at the site

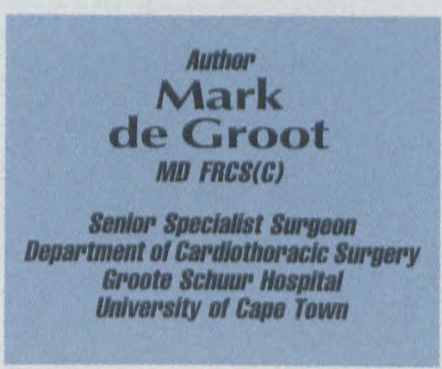

of intercostal retraction in the 5 th to 7 th intercostal spaces. These may be subtle on early films and may cause confusion with pathological fractures or tumour implants later in the clinical course if not recognised as a common occurrence.

Anaesthetists often facilitate resection by the intraoperative use of double lumen endotracheal tubes to produce single lung isolation. Most often they are introduced into the left mainstem bronchus so as not to impinge on the proximal right upper lobe orifice. Occasionally this is not possible and a right sided tube is used. Provisions are made in their design for a separate lobar orifice but due to the tenuous nature of this contact, right upper lobe collapse is a frequent observation immediately after a left pneumonectomy. Most of these changes are transient and few require intervention.

Elevation of the ipsilateral hemidiaphragm is normal post pneumonectomy. In the 50 s and 60 s surgeons often crushed the phrenic nerve with the mistaken concept that it facilitated the obliteration of the residual pleural space. The actual effect however was detrimental asit resulted in poor fixation of the mediastinum and interference with normal ventilation and coughing and has hence been abandoned. Pneumoperitoneum may occur with inadvertent penetration of the diaphragm. This is usually realised at the time and closed. If unrecognised, visceral herniation can occur and gas in herniated viscus may be mistaken for the normal post pneumonectomy appearance!.

The transgression of chest wall tissue planes at the time of surgery results in some degree of surgical emphysema in all postoperative radiographs. Unlike pulmonary lobectomy where continued air leak is common, a properly performed pneumonectomy is airtight, therefore any continuing accumulation may be indicative of a stump leak. Similarly if mediastinal or pericardial rents are created air may appear in these planes and is only rarely pathological ${ }^{2}$. In normal surgical practice a single chest drain is left in situ. In certain instances, such as in an uncomplicated intrapleural pneumonectomy, no chest drain need be left at all if the space is evacuated adequately before closure. Because of the possibility of occlusion by kinking or clot, the presence of a drain does not insure against over accumulation of air or fluid in the pleural space.

\section{Uncomplicated recovery}

Under normal circumstances, once evacuated, the pleural space immediately begins to disappear. The diaphragm elevates, the mediastinum shifts towards the ipsilateral side and to a lesser degree the ribs crowd together. The total effect is a volumetric reduction of $50-75 \%$. Theremaining air space, once the drains are removed, fills up with fluid by virtue of bleeding, weeping from lymphatics and passive transudation as air is resorbed ${ }^{1.3 .5}$. This fluid initially accumulates at a rate of about two interspaces a day.A drop in the fluid level greater than $2 \mathrm{~cm}$ at any time post surgery indicates the loss of fluid via thoracentesis, chest wall dehiscence, a diaphragm rent or 
trom page 5

bronchopleural fistula.Approximately $90 \%$ of the space will be filled by two weeks with complete obliteration at two to four months ${ }^{4}$. Ipsilateral mediastinal displacement is usual and this takes six to eight months to reach its maximum. The interruption of this normal progression usually heralds the development of empyema or other complications ${ }^{1}$. A postmortem series observed that in only one third of patients is there complete fibrous obliteration of this space even at many years post resection ${ }^{5}$. A typical course of events is illustrated in the series of radiographs in Figure 1. In early films, due to pain and splinting of the chest wall, it is typical to see an ipsilateral scoliosis. The remaining lung often appears to over-inflate in compensation which is due to the lower general intrathoracic pressure associated with mediastinal shift. In patients with chronic obstructive airways disease, hyperinflation may lead to transient deterioration in lung mechanics.

\section{Early Complications}

The most feared complication is the development of a bronchial stump leak which carries an overall mortality of about $15 \%$. If occurring acutely within the first 48 hours they are usually due to technical fault and require surgical reclosure. After this point it is often due to vascular stump necrosis or continuing infection ${ }^{1}$. The presence of residual tumour or active TB or infection increases the risk of this event. The resultant leak leads to an immediate bronchopleural fistula on the operative side which may culminate in a tension pneumothorax. In addition, inhalation of pleural contents from the pneumonectomy space soils the airways leading to a "drowning" of the patient's residual lung. The classic radiological appearance of this event is a dropping of the residual air-fluid level in operative space and a soiling of the remaininglung.

Another acute crisis is the development of cardiac herniation ${ }^{6.8}$.

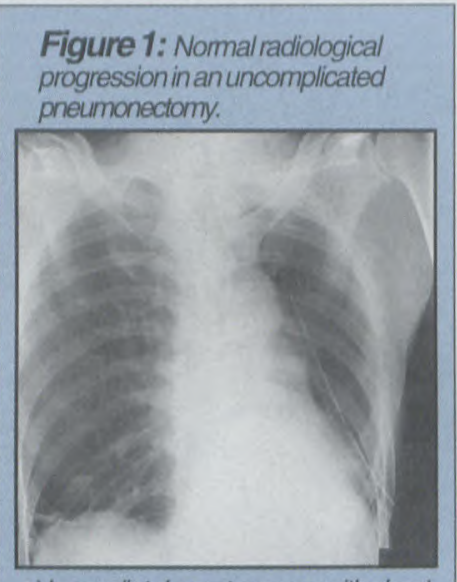

a) Immediately post surgery with chest drain in situ.

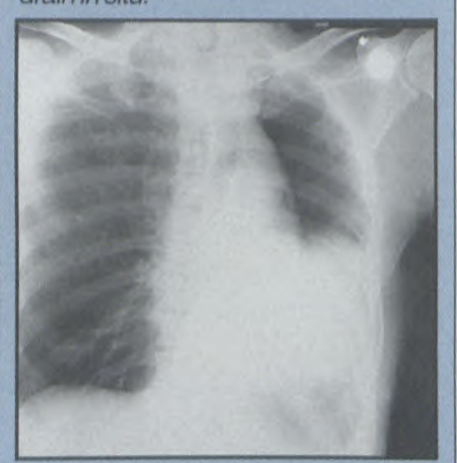

b) At 24 hours postoperation. Note the drain has been removedand the pleural space is beginning to fill with serum.

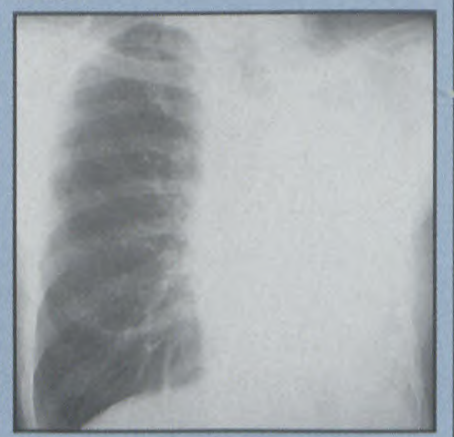

c) Sixmonths postoperation. The airspace has disappearedand the mediastinum has shifted towards the operativeside.

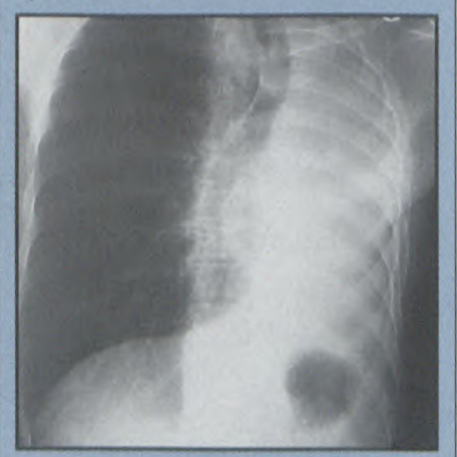

d) Twelvemonths postoperation. The mediastinum has further shifted and has reached its final position.
This occurs when by intent or accident a pericardial communication is created. Herniation usually occurs within the first few hours and may be precipitated by turning or repositioning the patient. In a right sided hernia, the heart rotates counterclockwise twisting the vena cavae. Radiologically the mediastinum is seen to be displaced to the right with the apex of the heart pointing to the right. The empty pericardial sac may be seen in the left hemithorax. With a left sided hernia the mediastinum shifts in that direction with a bulbous deviation in the left sided silhouette. Clinical findings include tachycardia, hypotension and elevated central venous pressure. Unless the heart is returned to normal position immediately the patient usually dies. Excessive or precipitous mediastinal shift may itself occlude venous return to the heart with haemodynamic consequence. This is usually caused by inappropriate application of suction to the pleural space or by the patient coughing or straining with an underwater seal drain connected. This ismore commonly seen in the paediatric population where the mediastinum is very pliable or the elderly where the shift needs to be less marked to produce a haemodynamic change. Unlike cardiac herniation the radiograph shows the mediastinum displaced as a unit with no contour changes.

Acute post pneumonectomy pulmonary oedema is a poorly understood phenomenon usually occurring in the first 24 hours post surgery and most commonly after a right pneumonectomy. It is thought to be due to movement of fluid across the interstitium of the lung caused by increased perfusion, alteration of myocardial performance and over transfusion ${ }^{9,10}$. Once developed it carries a mortality in excess of $50 \%$.

Over-accumulation of fluid in the pleural space may lead to contralateral mediastinal shift and respiratory embarrassment. In the early course this is typically due to bleeding but may also occur from over-transudation of plasma from osmotic effect from rents in the thoracic duct creating a tension chylothorax ${ }^{11-13}$. Where the pleural contents are contaminated an 


\section{AGFA Your growth path to an open future}

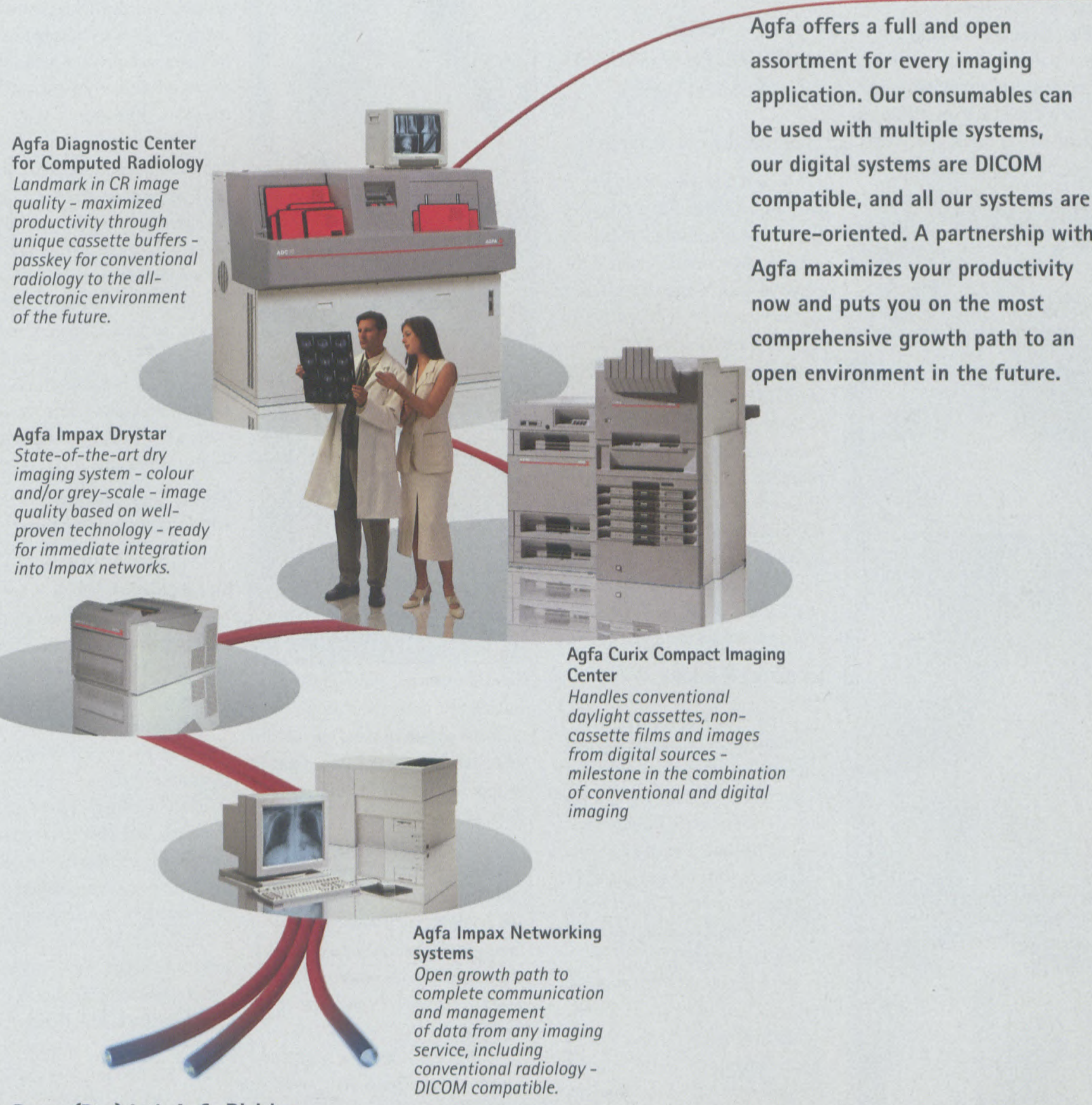

Bayer (Pty.) Ltd. Agfa Division

P.O. Box 143 Isando, 1600

Republic of South Africa

Tel.: (011) 9215911

Fax.: (011) 9215766

\section{Assure your future investments: choose Agfa.}


complication usually associated with surgery for inflammatory disease and almost exclusively in the right chest ${ }^{14}$. The morbid pathology is either direct damage to the viscus or its supplying vessels at the time of surgery. The clinical manifestations are indistinguishable from de novo empyema and the diagnosis is usually suspected only when food drains from the chest tube. Contrast studies are conclusive in nearly all cases. Subsequent surgery usually involves

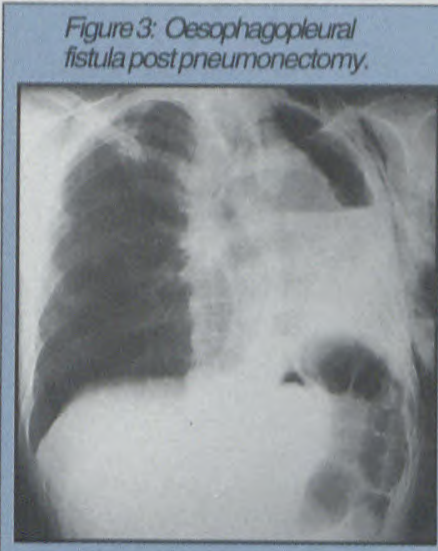

a) Radiograph taken on day 4 when the patient displayed signs of septicaemia.

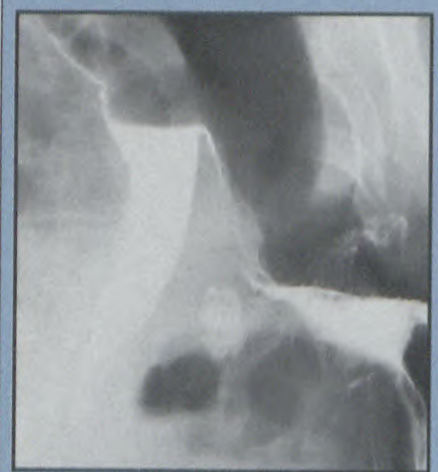

b) Confimation of cesophageal fistula by contrast swallow.

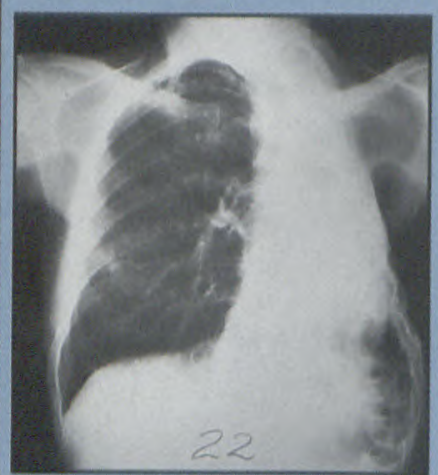

c) Finalappearance at6 monthisaftera thoracoplasty was performed to obliterate the fistula and close the space. (Note the scoliosis post thoracoplasty). the use of muscle flaps or thoracoplasty ${ }^{1,14}$ (Figure3). In unusual cases, this can occur many years after surgery and is thought to be due to internally pointing empyema from other sources.

\section{Late \\ Postoperative Changes}

\section{Late radiological changes}

When a pneumonectomy is performed before full pulmonary growth has occurred there may be compensatory development in the remaining lung. With associated chest wall development and mediastinal rotation this may lead to what appears to be herniation of the remaining lung into the contralateral chest. In extreme cases the situation may be difficult for the uninitiated to differentiate from the patienthaving bothlungsintact. (Figure 4). To a lesser degree this may occur in all patients having a pneumonectomyt. Radiological clues are the direction of the vessels and bronchi, the appearance of an unusual pleural line and the wide premediastinal space on thelateral $\mathrm{x}$-ray.

\section{Late complications}

With the resection of a lung for carcinoma it may be difficult to detect suspected intrathoracic recurrence of tumours on the standard radiograph due to obliteration of the interfaces between mediastinum, lung and chest wall. In these circumstances it may be necessary to resort to CT scanning or other imaging modalities to assist making the diagnosis ${ }^{15}$

Most patients will retain a small residual fluid filled space for the remainder of their life ${ }^{3}$. Either through haematogenous or direct contamination an empyema can develop at any time, even years, after surgery. The most common cause is stump breakdown which may be due to recurrent tumour, tuberculosis, or suture reaction. The empyema may "point"backinto the airway butcan also erode down the old incision or

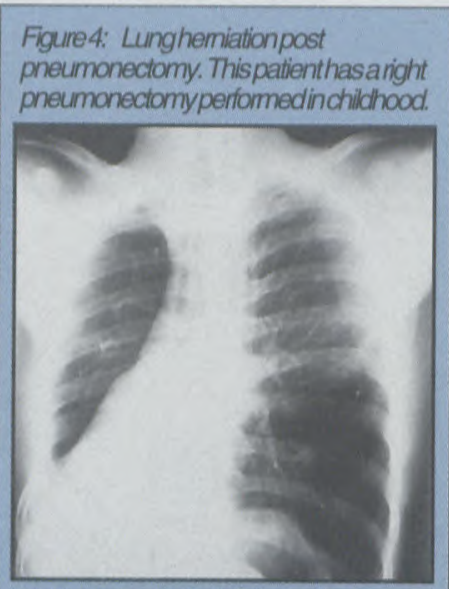

a) AP radiograph showing what appears to be residual right lung. (Note that vessels and bronchican be traced to left hilum).

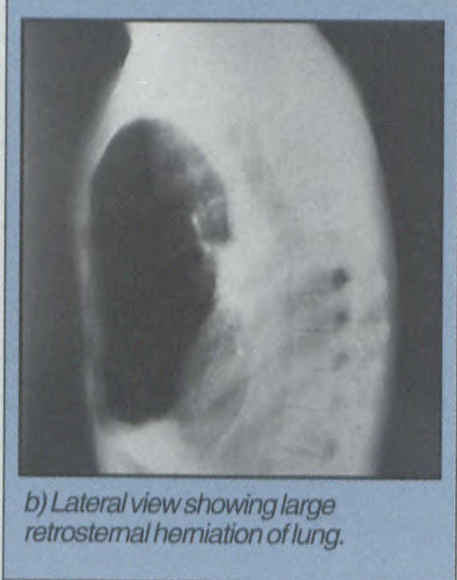

operative sites to appear on the skin surface. The necessitating empyema can point anywhere at any internal or superficial site and has even been noted to appear as far down as the groin. Radiologically these may be invisible until the partial drainage of contents leads to a visible airfluid level within the collection. Late bronchopleural fistula is found in about $50 \%$ of the former cases and when small and localised it may be difficult to detecteven with the use of CT scan or bronchographic contrast'.

Post pneumonectomy syndrome refers to airway obstruction secondary to resections in adolescence or childhood and also rarely in adults. Usually this occurs after a right pneumonectomy with the interval from pneumonectomy to development ranging from months to many years ${ }^{11,16}$. The aetiology is thought to be that the compliance of the mediastinum allows extreme rightward shift attenuating the left mainstem bronchus across the spine and between the aorta and pulmonary artery. Current treatment is either to place silicon or fluid filled prostheses within the pleural space to return the mediastinum to a neutral position ${ }^{17,18}$ or to splint the airways with endobronchial stents.

Long term elevation of the hemidiaphragm as seen routinely after pneumonectomy may be associated with the development of mesaxial and organoaxial malrotation of the stomach. This is likely to be seen in patients with eventration of the diaphragm and is very rare with few documented cases $^{19}$. Confirmation requires contrast studies and alleviation of symptoms by transabdominal fixation of the stomach.

\section{The radiograph after surgery for complications}

Complications particularly bronchopleural fistulas or post pneumonectomy empyema may have as part of their treatment the formation of a cutaneous stoma at the dependent part of the pleural space. Thisleads to a classical external "letter box" and a typical radiological appearance ${ }^{20,21}$ (Figure 5). After the creation of a stoma the pleural space may fibrose and obliterate over time. Alternatively, attempts at chemically sterilising the space and closing the flap may return the radiograph to a general post pneumonectomy appearance. Reticentcases are treated with muscle flap obliteration of the space $^{21,22}$ or thoracoplasty ${ }^{23}$ (Figure 5). Thoracoplasty remains as the definitive and often final procedure in a complicated post pneumonectomy course and is aimed at complete obliteration of the space. This tends to result in much superficial disfigurement and the development of scoliosis in many patients(Figure3). 


\section{The Post Pneumonectomy Chest Radiograph}

from page 8

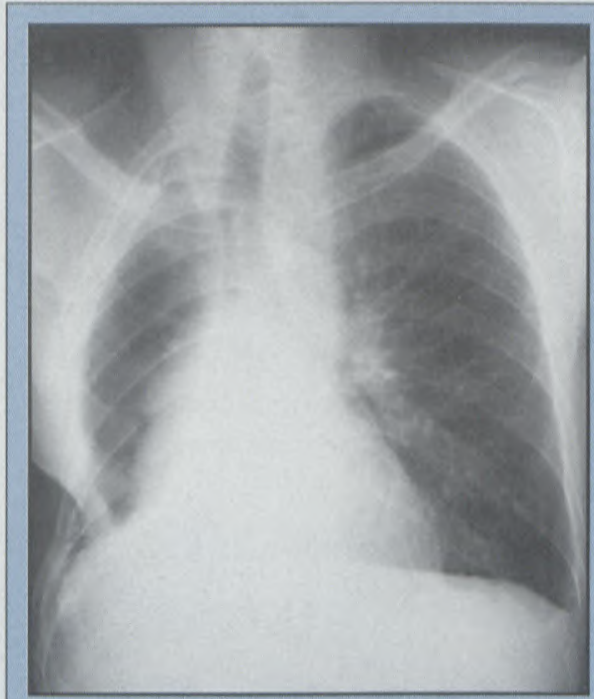

Figure 5: Open flap drain (Eloesser) of rightpost pneumonectomy empyema. The stoma is typically at the most dependent point and in property constructed cases no fluid levels should be seen presence or absence of complication. The challenge for the clinician is to have an appreciation of these changes as he is likely to encounter them in a lifelong clinical practice.

\section{References}

1. Spirn PW, Gross GW, Wechsler RI Steiner RM: Radiology of the chest after thoracic surgery. [Review]. Semin Roentgend 1988:23:931.

2.BrandenhoffP,Hoier:Madsen K, Struve-Christensen E: Pneumopericardium after pneumonectomy and lobectomy. Tharax 198641.55-57.

3.Silver AW,EspinasEE, Byron FX:Thefate of the posstresection space. Ann Thorac Surg 1966:2311-336

\section{Peculiarities}

One peculiarity of local surgery during the 60 s and 70 s was the transient use of intrapleural Ivalon sponge in an attempt to fibrose and obliterate the post pneumonectomy space. This was abandoned as it was subsequently shown to foster infection by acting as a permanent foreign body. The sponge "welded" itself to mediastinal structures rendering it unsafe to remove electively. The radiological appearance was quite classical (Figure 6) and patients still present many years later after successful surgery. Another transient peculiarity was the use of surgical wire for bronchial stump closure. This often has the patient presenting with recurrent minor haemoptysis due to persistent foreign body reaction and irritation. The diagnosis can frequently be made radiologically but should be confirmed via bronchoscopy to rule out more morbidcausation.

\section{Conclusion}

In conclusion, the post pneumonectomy radiograph can show a wide variation in appearance due to age, original diagnosis and the
4.Goodman LR: Postoperative chest radiograph: II. Alterations after major intrathoracic surgery. AJR Am J Roentgenol 1980,134:803813.

5.Suarez J, Clagett T, Brown AL, Jr. The postpmeumonectomy space:factors infulencing its obliteration. JThorac Cardiovasc Surg 1969:57:539542

6. Hidvegi RS, Abdulnour EM, Wilson IA: Herniation of the heart following left pneumonectomy. J Can Assoc Radiol 1981;32:185-187.

7. Chaukar AP, Swarup AS, Mandke NV Parulkar GB, Panday SR:Acute hemiation of Med1977:23:143-145.

8.DippelWF,Ehrenhaft IL:Hermiation of the heart after pneumonectomy. J Thorac CardiouascSung 1973,65:207-209.

9. Shapira OM, Shahian DM: Postpneumonectomy pulmonary edema.Ann ThoracSung 1993;56:190-195.

10. Mahfood S, HixWR, Aaron BL, BlaesP Watson DC:Reexpansion pulmonaryedema. AnmThorac Sung 1988:45:340-345.

11. Vallieres E, Shamji FM, Todd TR: Postpneumonectomy chylothorax. Ann Thorac Sung1993:55:1006-1008

12. Kanvande SV,WolcottMW, Gay WA, Jr: Postpmeumonectomytension chylothorax. Ann ThoracSung 1986:42:585-586.

13. Van Mulders A Lacquet LM, Van Mieghem W, Deneffe G: Chylothorax complicating pneumonectomy. Thorax 1984:39.954955.

14.Sethi GK, TakaroT:Esophagopleural fistula the heart following pneumonectomy. J Postgrad

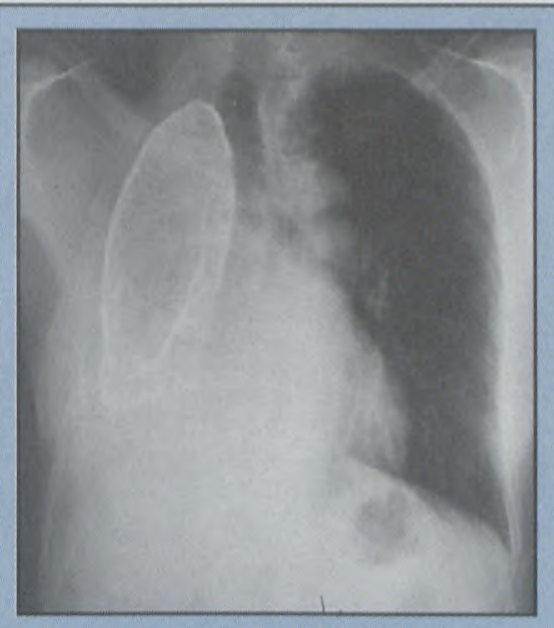

23. Gregoire R, Deslauriers J Beaulieu M, Piraux M: Thoracoplasty: its forgotten role in the management of nontuberculous postpneumonectomyempyema. CanJSung 1987;30:343-345.
Figure 6: Retainedforeign body post pneumonectomy. This patienthas right pneumonectomy performed in youth with the intentional placement of an Ivalon sponge to stimulate pleural fibrosis. This appearance is often confused with residual pleural calcification.

following pulmonary resection. Ann Thorac Sug 1978,25:7481.

15. GlazerHS, AronbergDJ,SagelSS, Emami B: Utility of $C T$ in detecting postpneumonectomy carcinoma recurrence. A/RAm JReentgenol 1984; 142:487-494.

16. Grillo HC, Shepard JA, Mathisen DJ, KanarekDJ:Postpneumonectomysyndrome: diagnosis, management, and results. Ann ThonacSung1992;54.63850; disaussion6501.

17.Rivern FA,Adams C,Lewis $J W_{1} \mathrm{Jr}, \mathrm{O}$. hs D, Glines C, Popovich J,Jr.Silastic prosthesis plombage for right postpneumonectomy syndrome. Ann Thorac Surg 1990;50:465466

18. Kosloske AM, Williamson SL: An expandable prosthesisfor stabilization of the infantmediastimum followingpneumonedomy. $J$ PedianSiog1992:27:1521-1522.

19. CantisleBB,Hayes CW:Gastricuobulus An unusual complication after pneumonectomv. Am JSung 1967;113:579. 582

20. ShapiroMP,GaleME, Daly BD:Eloesser window thoracostomy for treatment of empyema: radiographic appearance. AJR AmJRoentgenol 1988;150:549-552.

21. Amive L, Tasu JP, Kitzis M, etal:Open window thoracostomy, myoplasty, and epiploplasty for treatment of postpneumonectomy empyema: CT evaluation Radiology 1994;192:177-181.

22. Bhalla M, Wain JC, Shepard JA, McLoud TC: Surgical flaps in the chest: anatomic considerations, applications, and radiologic appearance. Radiology 1994:192:825:830. 